\title{
Lymph node metastases in resected pancreatic ductal adenocarcinoma: predictors of disease recurrence and survival
}

Vicente Morales-Oyarvide 1,13, Douglas A Rubinson 1,13, Richard F Dunne ${ }^{2}$, Margaret M Kozak ${ }^{3}$, Justin L Bui ${ }^{3}$, Chen Yuan ${ }^{1,4}$, Zhi Rong Qian ${ }^{1}$, Ana Babic ${ }^{1}$, Annacarolina Da Silva ${ }^{1}$, Jonathan A Nowak ${ }^{5}$, Natalia Khalaf', Lauren K Brais ${ }^{1}$, Marisa W Welch ${ }^{1}$, Caitlin L Zellers ${ }^{1}$, Kimmie Ng ${ }^{1}$, Daniel T Chang ${ }^{3}$, Rebecca A Miksad ${ }^{7}$, Andrea J Bullock ${ }^{7}$, Jennifer F Tseng ${ }^{8}$, Richard S Swanson ${ }^{9}$, Thomas E Clancy ${ }^{9}$, David C Linehan ${ }^{10}$, Jennifer J Findeis-Hosey ${ }^{11}$, Leona A Doyle ${ }^{5}$, Jason L Hornick ${ }^{5}$, Shuji Ogino ${ }^{1,4,5}$, Charles S Fuchs ${ }^{1}$, Aram F Hezel ${ }^{2}$, Albert C Koong ${ }^{12}$ and Brian M Wolpin ${ }^{*, 1}$

${ }^{1}$ Department of Medical Oncology, Dana-Farber Cancer Institute and Harvard Medical School, 450 Brookline Avenue, Boston, MA 02215, USA; ${ }^{2}$ Department of Medicine, Division of Hematology and Oncology, Wilmot Cancer Institute, University of Rochester Medical Center, 601 Elmwood Avenue, Rochester, NY 14642, USA; ${ }^{3}$ Department of Radiation Oncology, Stanford Cancer Institute, 269 Campus Drive West, Stanford, CA 94305-5152, USA; ${ }^{4}$ Department of Epidemiology, Harvard TH Chan School of Public Health, 677 Huntington Avenue, Boston, MA 02115, USA; ${ }^{5}$ Department of Pathology, Brigham and Women's Hospital and Harvard Medical School, 75 Francis Street, Boston, MA 02115, USA; ${ }^{6}$ Division of Gastroenterology, Hepatology, and Endoscopy, Brigham and Women's Hospital, 75 Francis Street, Boston, MA 02115, USA; ${ }^{7}$ Department of Hematology and Oncology, Beth Israel Deaconess Medical Center and Harvard Medical School, 330 Brookline Avenue, Boston, MA 02215, USA; ${ }^{8}$ Department of Surgery, Beth Israel Deaconess Medical Center and Harvard Medical School, 330 Brookline Avenue, Boston, MA 02215, USA; ${ }^{9}$ Department of Surgery, Brigham and Women's Hospital and Harvard Medical School, 75 Francis Street, Boston, MA 02115, USA; ${ }^{10}$ Department of Surgery, University of Rochester Medical Center, 601 Elmwood Avenue, Rochester, NY 14642, USA; ${ }^{11}$ Department of Pathology, University of Rochester Medical Center, 601 Elmwood Avenue, Rochester, NY 14642, USA and ${ }^{12}$ Department of Radiation Oncology, The University of Texas MD Anderson Cancer Center, 1840 Old Spanish Trail, Houston, TX 77054, USA

Background: Few studies have simultaneously assessed the prognostic value of the multiple classification systems for lymph node (LN) metastases in resected pancreatic ductal adenocarcinoma (PDAC).

Methods: In 600 patients with resected PDAC, we examined the association of LN parameters (AJCC 7th and 8th editions, LN ratio (LNR), and log odds of metastatic LN (LODDS)) with pattern of recurrence and patient survival using logistic regression and Cox proportional hazards regression, respectively. Regression models adjusted for age, sex, margin status, tumour grade, and perioperative therapy.

Results: Lymph node metastases classified by AJCC 7th and 8th editions, LNR, and LODDS were associated with worse disease freesurvival (DFS) and overall survival (OS) (all $P_{\text {trend }}<0.01$ ). American Joint Committee on Cancer 8th edition effectively predicted DFS and OS, while minimising model complexity. Lymph node metastases had weaker prognostic value in patients with positive margins and distal resections (both $P_{\text {interaction }}<0.03$ ). Lymph node metastases by AJCC 7th and 8th editions did not predict the likelihood of local disease as the first site of recurrence.

Conclusions: American Joint Committee on Cancer 8th edition LN classification is an effective and practical tool to predict outcomes in patients with resected PDAC. However, the prognostic value of LN metastases is attenuated in patients with positive resection margins and distal pancreatectomies.

${ }^{*}$ Correspondence: Dr BM Wolpin; E-mail: bwolpin@partners.org

${ }^{13}$ Co-first authors.

Received 14 April 2017; revised 16 August 2017; accepted 7 September 2017; published online 5 October 2017

(C) 2017 Cancer Research UK. All rights reserved 0007-0920/17 
Pancreatic cancer is the third leading cause of cancer death in the United States (Siegel et al, 2016). Regional lymph node (LN) involvement is an important predictor of survival in patients undergoing resection for pancreatic cancer (Lim et al, 2003; Richter et al, 2003; Schwarz and Smith, 2006; Winter et al, 2006). The current standard for pancreatic cancer staging is the American Joint Committee on Cancer (AJCC) 7th edition system that classifies nodal involvement as pN0 if no regional LN metastases are present or $\mathrm{pN} 1$ when regional $\mathrm{LN}$ metastases are identified (Edge et al, 2010).

Recent studies have suggested that the number of metastatic LN provide additional prognostic information (Murakami et al, 2007; Basturk et al, 2015; Malleo et al, 2015; Strobel et al, 2015). Therefore, the upcoming 8th edition of the AJCC system will stratify LN-positive patients into pN1 (metastases in 1-3 regional $\mathrm{LN}$ ) and pN2 (metastases in $\geqslant 4$ regional LN) (Allen et al, 2017). Several studies in pancreatic cancer and other malignancies have suggested that prognosis is associated with the relationship of metastatic-to-examined LN, as assessed by the LN ratio (LNR) and log of the odds of metastatic LN (LODDS) (Slidell et al, 2008; Valsangkar et al, 2013; La Torre et al, 2014; Riediger et al, 2016). Simultaneous investigations of these different LN parameters in relation to patient outcomes are lacking, particularly in large, multi-institutional patient cohorts. How these LN parameters predict patterns of disease recurrence (e.g., local vs distant recurrence) is not well understood, yet has important implications for perioperative treatment programmes. Recent clinical practice guidelines from the American Society of Clinical Oncology (ASCO) recommend the use of adjuvant radiation in patients with LN metastases at resection (Khorana et al, 2016). In contrast, current National Comprehensive Cancer Network guidelines for treatment of pancreatic cancer do not take LN status into consideration when recommending for or against radiation treatment in the adjuvant setting (National Comprehensive Cancer Network). Moreover, the number of examined LN and presence of lymphovascular invasion have been reported as prognostic factors particularly in patients with $\mathrm{LN}$-negative disease (Hellan et al, 2008; Takahashi et al, 2012; Valsangkar et al, 2013), raising the question of whether these factors influence outcomes differently in patients with LN-negative and -positive tumours.

The primary aim of the current study was to evaluate the ability of different LN classification systems to predict disease recurrence and survival in a large series of patients with resected pancreatic ductal adenocarcinomas (PDAC) from multiple US centres. As a secondary goal, we also sought to determine whether LN involvement predicts pattern of disease recurrence following resection.

\section{MATERIALS AND METHODS}

Study population. The initial patient population consisted of 600 patients who underwent surgical resection for PDAC at academic and community hospitals in the United States. Surgery was performed at Dana-Farber/Brigham and Women's Cancer Center (DF/BWCC, Boston, MA, USA; $n=274$ ) between October 2002 and June 2015, at 35 academic and community hospitals between September 2000 and June 2015 referred to the DFCI/BWCC for a second opinion ( $n=87$; list of hospitals in Supplementary Table 1), at the University of Rochester Medical Center (URMC, Rochester, NY, USA; $n=90$ ) between 1 March 2006 and 1 November 2013, and at Stanford Cancer Institute (SCI, Stanford, CA, USA; $n=149$ ) between September 1995 and May 2013. Institutional Review Board (IRB) approval was granted at DF/BWCC, URMC, and SCI. Patients who received care at DF/BWCC signed informed consent for participation in this study. For those receiving treatment at
URMC and SCI, informed consent was waived as patients were identified retrospectively on Institutional Review Board exempt protocols.

Assessment of LN parameters. The AJCC (7th edition) system classifies LN involvement as pN0 (no regional LN metastases), pN1 (regional LN metastases), or $\mathrm{pNx}$ (regional LN cannot be assessed) (Edge et al, 2010). The upcoming AJCC (8th edition) system classifies LN involvement as pN0 (no regional LN metastases), pN1 (metastases in $1-3$ regional $\mathrm{LN}$ ), pN2 (metastases in $\geqslant 4$ regional $\mathrm{LN}$ ), or $\mathrm{pNx}$ (regional LN cannot be assessed) (Allen et al, 2017). lymph node ratio was calculated as (no. of positive LN/ no. of examined LN) and classified into four categories: 0, 0.01-0.20, 0.21-0.40, and >0.40 (Malleo et al, 2015; Strobel et al, 2015). Log odds of metastatic LN was calculated as ((no. of positive LN + 0.5)/ ( no. of negative $\mathrm{LN}+0.5)$ ), where 0.5 is added in the numerator and denominator to avoid null and undefined values, and then transformed to natural logarithmic scale (La Torre et al, 2014; Riediger et al, 2016). We explored the number of examined LN and LVI in secondary analyses.

Assessment of covariates. We collected data from medical records, operative notes, and pathology reports. We assessed sex, age, type of pancreatectomy, perioperative systemic and radiation treatment, tumour location, AJCC (7th and 8th editions) pT stage, tumour grade, resection margin status, LVI, and perineural invasion. Resection margins were classified as R0 (no tumour cells within $1 \mathrm{~mm}$ of any pathologic margin), R1 (microscopic foci of tumour cells within $1 \mathrm{~mm}$ of a pathologic margin), R2 (gross residual disease), or $\mathrm{Rx}$ (margins not evaluable). For patients referred to DF/BWCC after undergoing surgery at another institution, slides were rereviewed by DF/BWCC gastrointestinal pathologists. The above covariates were considered for multivariable-adjusted model building as detailed below.

Outcome measures. Disease-free survival (DFS) was defined as time between date of surgery and date of disease recurrence. For patients who received neoadjuvant therapy, follow-up started on the date preoperative treatment was initiated. Patients who were alive and free of disease at the end of follow-up and those who died without definitive evidence of recurrence were censored for DFS analysis on the date of last clinical contact. Overall survival (OS) was defined as time between date of surgery (or date of start of neoadjuvant treatment) and date of death from any cause. Pattern of first recurrence was classified as 'local only' (recurrence in/or adjacent to the pancreatic remnant, retroperitoneum and periadventitial tissues near the resected pancreas), 'distant only' (recurrence only outside areas defined by local recurrence), or 'synchronous local and distant' (evidence of local and distant disease). Follow-up continued through 28 June 2016 for DF/BWCC patients, 17 March 2016 for URMC, and 11 March 2016 for SCI.

Case selection. Supplementary Figure 1 illustrates the derivation of the patient populations for outcome and recurrence analyses. We excluded patients found to have metastatic spread at the time of resection $(n=10), \mathrm{pNx}$ status $(n=3)$, and patients who died within 30 days after surgery or during initial hospitalisation $(n=13)$.

Statistical analysis. Associations between LN parameters, DFS, and OS were evaluated using multivariable-adjusted Cox proportional hazards regression models calculating hazard ratios (HRs) and $95 \%$ confidence intervals (CIs). To build the multivariableadjusted model, we used a training set consisting of subjects who received treatment at DF/BWCC $(n=361)$. We identified covariates for inclusion in the final model by performing stepwise selection with Cox proportional hazards regression using 'entry' and 'keep' thresholds of $P \leqslant 0.2$ and $P \leqslant 0.1$, respectively. With 
these parameters, we fitted a model that included six covariates: age at the time of surgery, sex, tumour grade (well/moderately differentiated, poorly differentiated/undifferentiated, unknown), perioperative systemic chemotherapy (yes, no), perioperative radiation treatment (yes, no), and resection margin status (R0, R1, R2, Rx). Proportionality of hazards assumption was satisfied by evaluating a time-dependent variable of the cross-product of the exposure of interest and time.

Using the above model, we compared the LN classification parameters by measuring model discrimination and calibration in the validation set, which consisted of subjects who received care at the URMC and SCI $(n=239)$. We evaluated model discrimination for each LN parameter using the overall C index - an extension of the area under a receiver-operator curve to survival analysis - and calculated its 95\% CI (Pencina and D'Agostino, 2004). Model calibration for each LN parameter was assessed using the Greenwood-Nam-D’Agostino goodness-of-fit test, where $P<0.05$ indicates model miscalibration (Demler et al, 2015).

As neoadjuvant treatment may affect LN status and the number of examined LN, we conducted sensitivity analyses restricted to patients who did not receive neoadjuvant therapy in the training set. We also performed stratified analyses by resection margin status (R0 or R1) and type of pancreatectomy (pancreaticoduodenectomy or distal resection). Interaction was assessed by including the cross-product term of the exposure and stratification variable in the regression model. Given that sample sizes were reduced in stratified analyses and similar associations were identified in the training and validation sets, these analyses were conducted in the entire study population (i.e., training and validation sets combined) and were further adjusted for the institution where patients received care (DF/BWCC, URMC, or SCI). Survival was also shown and assessed using Kaplan-Meier curves and log-rank tests in the entire study population.

Associations between LN parameters and pattern of first recurrence were analysed with logistic regression calculating odds ratios (ORs) and 95\% CI in the entire study population. Logistic regression models included the same covariates as in the Cox models described above. We also performed stratified analyses by receipt of perioperative radiation treatment.

We conducted linear trend tests across categories of exposure variables by assigning each subject the median value for the exposure category and modelling it as a continuous variable in Cox or logistic regressions. As we evaluated four LN classification parameters in our main analyses, statistical significance was adjusted for multiple comparisons and set at $P<0.013$. All hypothesis tests were two sided and analyses were performed using the SAS Software (version 9.4; SAS Institute, Cary, NC, USA).

\section{RESULTS}

Baseline characteristics of the 600 patients in the initial study population are shown in Table 1. At the end of follow-up, 173 (30.4\%) patients were alive and 108 (19.4\%) were alive without recurrent disease. Median follow-up time among patients who were alive at the end of follow-up was 26.4 months. In our study population, median DFS and OS were 14.3 and 22.5 months, respectively, and 5-year DFS and OS were $14.1 \%$ and $15.4 \%$, respectively, comparable to recent randomised trials of pancreatectomy followed by adjuvant therapy (Supplementary Table 2) (Oettle et al, 2007, 2013; Regine et al, 2008, 2011; Neoptolemos et al, 2010, 2017). As noted in other contemporary patient populations with resected PDAC (Oettle et al, 2007; Neoptolemos et al, 2010), nearly two in three patients had LN metastases.
We first examined the association of LN parameters with DFS and OS in the training set (Table 2) and found that higher categories of LN involvement were significantly associated with worse outcomes. We next evaluated the LN parameters' prognostic performance in the validation set, noting similar associations to those identified in the training set (Table 3). Lymph node involvement as classified by the 7th and 8th editions of the AJCC system was significantly associated with poor outcomes. Lymph node ratio and LODDS were associated with a stepwise increase in the hazards for recurrence ( $P_{\text {trend }}=0.004$ and 0.005 , respectively). The overall $C$ index was similar for the four LN parameters, indicating good discrimination without demonstrating superiority of one parameter over the others. Likewise, the Greenwood-NamD'Agostino goodness-of-fit test indicated adequate calibration for all LN parameters (all $P>0.60$ ). Kaplan-Meier survival curves of DFS and OS for each LN parameter in the entire study population are presented in Figure 1 and Supplementary Figure 2. Associations were largely unchanged after excluding the $14 \%$ of patients who received neoadjuvant therapy from the training set (Supplementary Table 3).

A recent validation study of AJCC (8th edition) classification was restricted to patients with R0 resections (Allen et al, 2017). Therefore, we assessed whether the relationship of LN parameters with survival differed by resection margin status (Table 4). We found that associations of AJCC (7th edition) and AJCC (8th edition) $\mathrm{pN}$ status with DFS were seen primarily in patients who underwent R0 resections, with less prognostic ability in patients with R1 margins $\left(P_{\text {interaction }}<0.02\right)$.

Given that anatomical differences influence surgical LN dissection, we also evaluated whether the association between LN parameters and survival differed by resection type. Lymph node metastases classified by AJCC (7th edition) and AJCC (8th edition) were significantly associated with disease recurrence and mortality among patients who underwent pancreaticoduodenectomy, but not among patients who underwent distal resection (Table 4). Pancreaticoduodenectomy specimens had higher number of examined (median, $16 \mathrm{LN}$ vs $12.5 \mathrm{LN}, P<0.01$ ) and positive $\mathrm{LN}$ (median, $2 \mathrm{LN}$ vs $0 \mathrm{LN}, P<0.01$ ) compared with distal resections.

We next assessed the number of examined LN and presence of LVI as markers of disease recurrence and survival (Supplementary Table 4). Fewer than 12 LN examined was not associated with DFS or OS among LN-negative or -positive patients. In contrast, the presence of LVI revealed a trend towards poor DFS in patients with LN-negative (HR 1.50; 95\% CI: 0.90-2.51) and LN-positive (HR 1.27; 95\% CI: $0.96-1.67)$ disease $\left(P_{\text {interaction }}=0.783\right)$.

Recent ASCO clinical practice guidelines recommend consideration of adjuvant chemoradiation for patients with R1 resections and/or LN-positive tumours (Khorana et al, 2016). Since the primary aim of chemoradiation is achieving local control, we assessed whether LN involvement predicts local recurrence (Table 5). In our study population, AJCC ( 7 th edition) and AJCC (8th edition) LN status did not predict local recurrence as first site of failure. Nevertheless, $41.8 \%$ of patients received perioperative radiotherapy that could influence their patterns of disease recurrence. Therefore, we conducted stratified analyses based on receipt of perioperative radiation (Supplementary Table 5). Although somewhat limited by sample size, LN metastases were not predictive for local disease as first site of recurrence even among patients who received no perioperative radiation.

\section{DISCUSSION}

In the current study, we found that LN categorisation by AJCC (7th edition), AJCC (8th edition), LNR, and LODDS all predicted 
Table 1. Baseline characteristics of 600 patients with resected pancreatic ductal adenocarcinoma

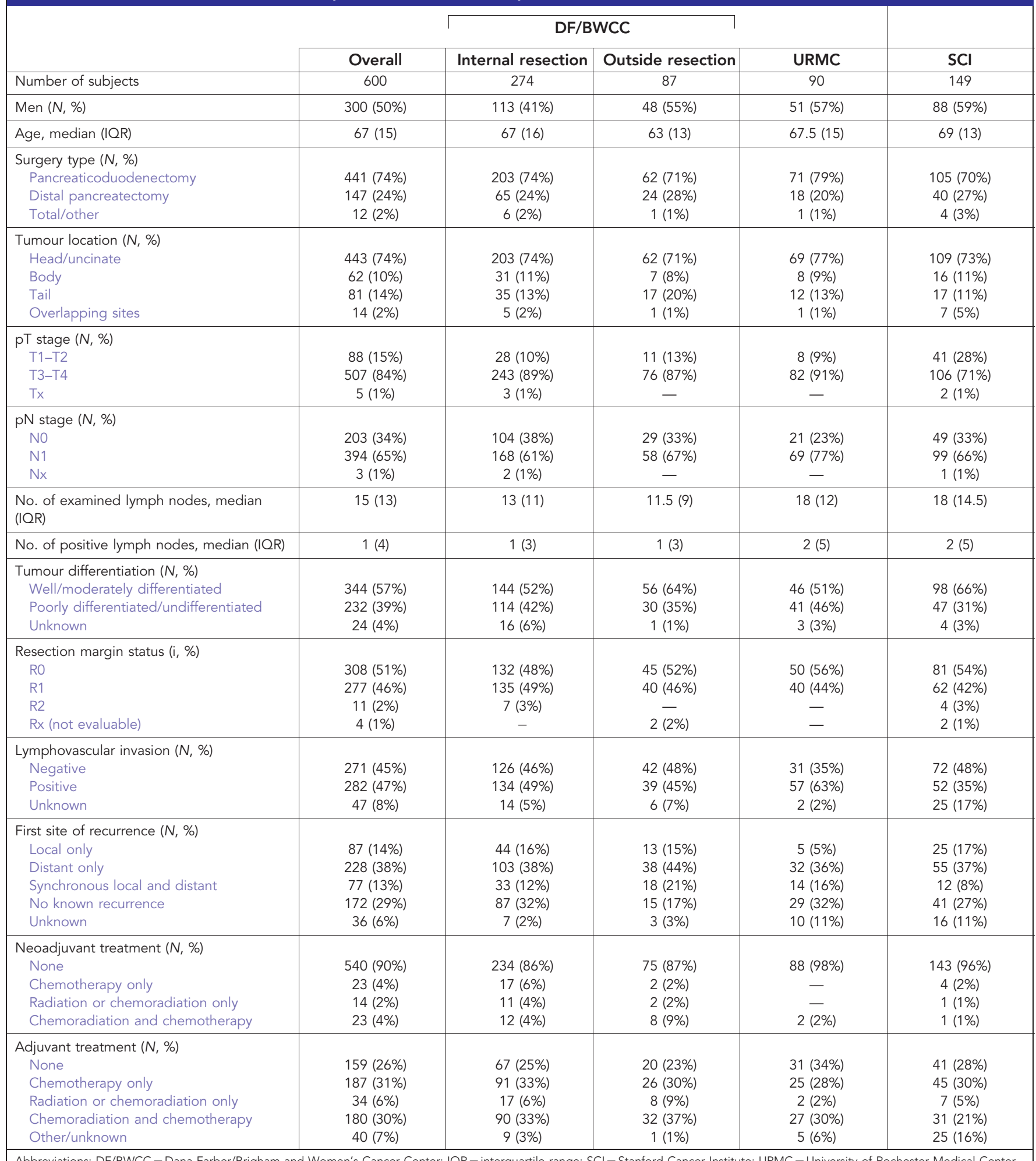

Abbreviations: DF/BWCC = Dana-Farber/Brigham and Women's Cancer Center; IQR = interquartile range; SCI=Stanford Cancer Institute; URMC=University of Rochester Medical Center.

DFS and OS in patients undergoing resection for PDAC. The AJCC (8th edition) provided good model fit, while minimising model complexity compared with LNR and LODDS. The prognostic value of LN metastases was stronger in patients with $\mathrm{R} 0$ resections and those who underwent pancreaticoduodenectomy, with weaker prognostic ability in patients with microscopically positive or close margins (R1) or patients with distal resections. Lymph node metastases did not predict local disease recurrence as first site of failure, even among patients who received no perioperative radiation. The presence of LVI was marginally associated with adverse outcomes in patients with both LNnegative and -positive tumours, while the number of LN examined was not prognostic in either group. These findings are relevant to prognostication for patients, while suggesting that positive LN status may not be useful in selecting patients for adjuvant radiotherapy. 
Table 2. DFS and OS by lymph node classification: training set

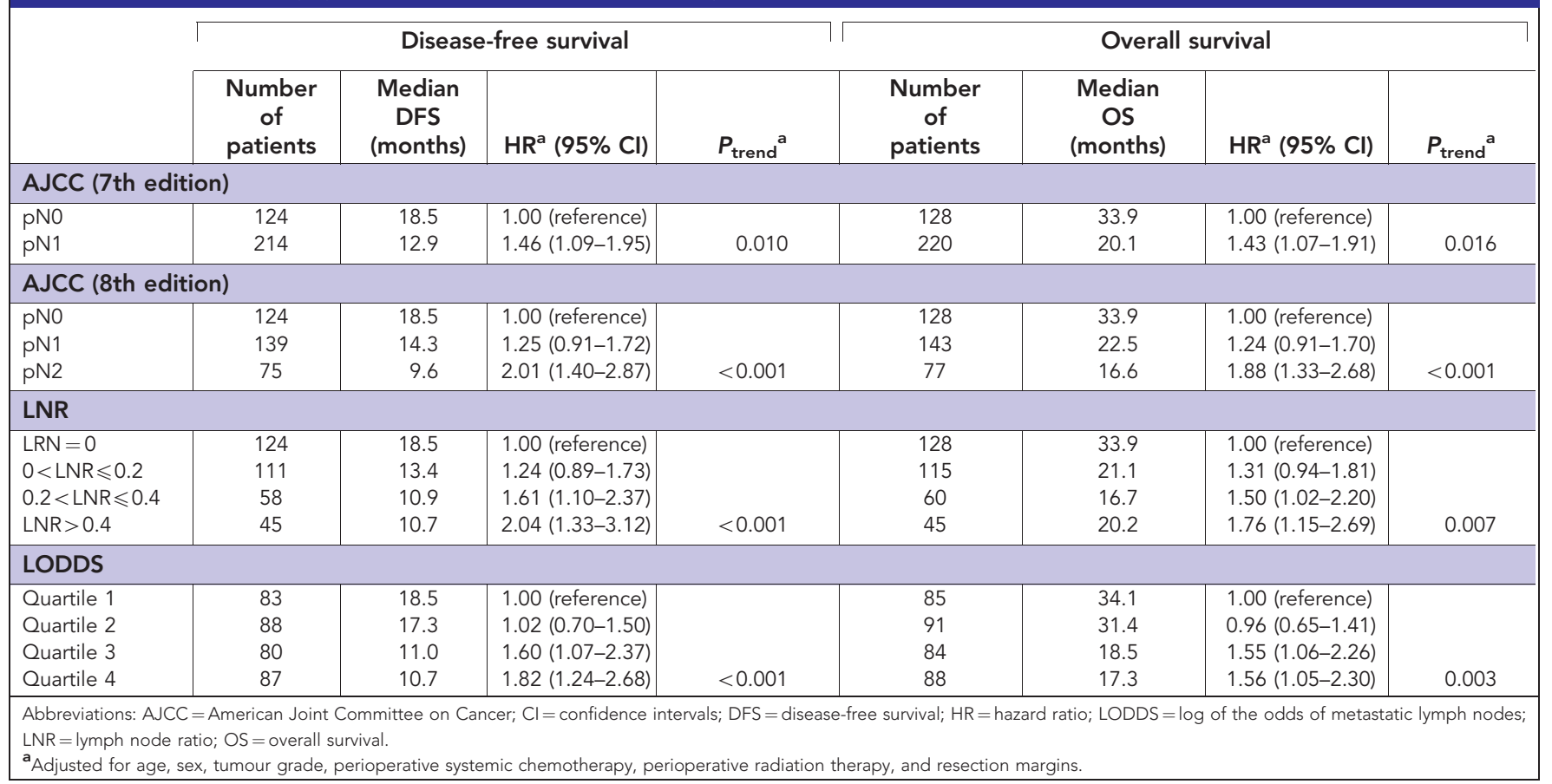

Table 3. DFS and OS by lymph node classification: validation set

\begin{tabular}{|c|c|c|c|c|c|c|c|c|c|c|c|c|}
\hline & \multicolumn{6}{|c|}{ Disease-free survival } & \multicolumn{6}{|c|}{ Overall survival } \\
\hline & $\begin{array}{c}\text { Number } \\
\text { of } \\
\text { patients }\end{array}$ & $\begin{array}{c}\text { Median } \\
\text { DFS } \\
\text { (months) }\end{array}$ & $\mathrm{HR}^{\mathrm{a}}(95 \% \mathrm{Cl})$ & $P_{\text {trend }}{ }^{a}$ & $\begin{array}{l}C \text { index } \\
(95 \% \mathrm{Cl})\end{array}$ & $P_{\mathrm{GND}}{ }^{\mathrm{c}}$ & $\begin{array}{c}\text { Number } \\
\text { of } \\
\text { patients }\end{array}$ & $\begin{array}{c}\text { Median } \\
\text { OS } \\
\text { (months) }\end{array}$ & $\mathrm{HR}^{\mathrm{a}}(95 \% \mathrm{Cl})$ & $P_{\text {trend }}{ }^{a}$ & $\begin{array}{l}C \text { index } \\
(95 \% \mathrm{Cl})\end{array}$ & $P_{\text {GND }}{ }^{\mathrm{c}}$ \\
\hline \multicolumn{13}{|c|}{ AJCC (7th edition) } \\
\hline $\begin{array}{l}\mathrm{pN} 0 \\
\mathrm{pN} 1\end{array}$ & $\begin{array}{r}62 \\
158\end{array}$ & $\begin{array}{l}27.8 \\
10.8\end{array}$ & $\begin{array}{l}1.00 \text { (reference) } \\
2.59(1.68-4.00)\end{array}$ & $<0.001$ & $\begin{array}{c}0.77 \\
(0.61-0.91)\end{array}$ & 0.75 & $\begin{array}{l}64 \\
158\end{array}$ & $\begin{array}{l}42.3 \\
17.4\end{array}$ & $\begin{array}{l}1.00 \text { (reference) } \\
2.90(1.87-4.48)\end{array}$ & $<0.001$ & $\begin{array}{c}0.79 \\
(0.63-0.91)\end{array}$ & 0.79 \\
\hline \multicolumn{13}{|c|}{ AJCC (8th edition) } \\
\hline $\begin{array}{l}\mathrm{pN} 0 \\
\mathrm{pN} 1 \\
\mathrm{pN} 2\end{array}$ & $\begin{array}{l}62 \\
77 \\
81\end{array}$ & $\begin{array}{l}27.8 \\
10.8 \\
11.0\end{array}$ & $\begin{array}{l}1.00 \text { (reference) } \\
2.60(1.63-4.14) \\
2.58(1.58-4.21)\end{array}$ & 0.001 & $\begin{array}{c}0.77 \\
(0.61-0.91)\end{array}$ & 0.83 & $\begin{array}{l}64 \\
77 \\
81\end{array}$ & $\begin{array}{l}42.3 \\
16.9 \\
17.4\end{array}$ & $\begin{array}{l}1.00 \text { (reference) } \\
2.79(1.75-4.46) \\
3.03(1.87-4.93)\end{array}$ & $<0.001$ & $\begin{array}{c}0.79 \\
(0.63-0.91)\end{array}$ & 0.62 \\
\hline \multicolumn{13}{|l|}{ LNR } \\
\hline $\begin{array}{l}L R N=0 \\
0<L N R \leq 0.2 \\
0.2<L N R \leq 0.4 \\
L N R>0.4\end{array}$ & $\begin{array}{l}62 \\
76 \\
53 \\
29\end{array}$ & $\begin{array}{r}27.8 \\
13.1 \\
10.7 \\
6.6\end{array}$ & $\begin{array}{l}1.00 \text { (reference) } \\
2.52(1.59-4.00) \\
2.73(1.56-4.78) \\
2.69(1.45-5.01)\end{array}$ & 0.004 & $\begin{array}{c}0.77 \\
(0.61-0.91)\end{array}$ & 0.94 & $\begin{array}{l}64 \\
76 \\
53 \\
29\end{array}$ & $\begin{array}{l}42.3 \\
18.4 \\
16.0 \\
14.8\end{array}$ & $\begin{array}{l}1.00 \text { (reference) } \\
2.64(1.64-4.24) \\
3.36(2.01-5.63) \\
3.04(1.68-5.53)\end{array}$ & $<0.001$ & $\begin{array}{c}0.78 \\
(0.63-0.91)\end{array}$ & 0.68 \\
\hline \multicolumn{13}{|l|}{ LODDS } \\
\hline $\begin{array}{l}\text { Quartile } 1 \\
\text { Quartile } 2 \\
\text { Quartile } 3 \\
\text { Quartile } 4\end{array}$ & $\begin{array}{l}54 \\
57 \\
56 \\
53\end{array}$ & $\begin{array}{r}20.0 \\
14.8 \\
10.8 \\
9.7\end{array}$ & $\begin{array}{l}1.00 \text { (reference) } \\
1.83(1.10-3.04) \\
1.64(0.96-2.81) \\
2.37(1.36-4.12)\end{array}$ & 0.005 & $\begin{array}{c}0.78 \\
(0.61-0.91)\end{array}$ & 0.98 & $\begin{array}{l}54 \\
58 \\
57 \\
53\end{array}$ & $\begin{array}{l}49.8 \\
\\
20.0 \\
15.8 \\
14.8\end{array}$ & $\begin{array}{l}1.00 \text { (reference) } \\
2.21(1.27-3.85) \\
3.04(1.78-5.19) \\
3.44(1.96-6.05)\end{array}$ & $<0.001$ & $\begin{array}{c}0.79 \\
(0.64-0.91)\end{array}$ & 0.74 \\
\hline \multicolumn{13}{|c|}{ 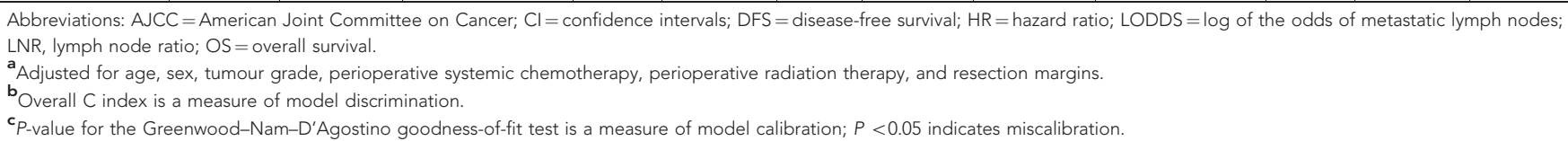 } \\
\hline
\end{tabular}

Recent studies have suggested that the number of positive LN may improve prognostication over the binary designation of negative vs positive LN involvement (Murakami et al, 2007; Basturk et al, 2015; Malleo et al, 2015; Strobel et al, 2015). Consequently, the new AJCC (8th edition) staging of pancreatic cancer will distinguish patients with 1-3 metastatic LN from those with $\geqslant 4$ metastatic LN (Allen et al, 2017). In this study, we confirmed the additional prognostic benefit to this approach, with median OS times of 35.1, 20.6, and 16.8 months in the entire study population for patients with N0, N1, and N2 disease, respectively. Importantly for clinicians, the value of either AJCC nodal classification in predicting patient outcomes was stronger among patients with margin-negative resections and those with proximal tumours undergoing pancreaticoduodenectomy. The prognostic 
A

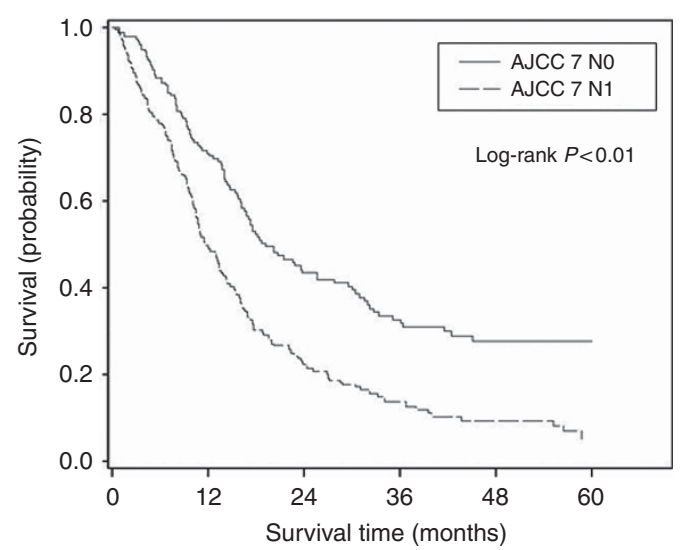

No. at risk

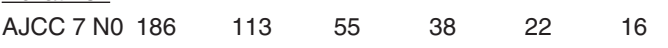

$\begin{array}{llllll}\text { AJCC } 7 \text { N1 } 372 & 143 & 51 & 23 & 9 & 3\end{array}$

\section{C}

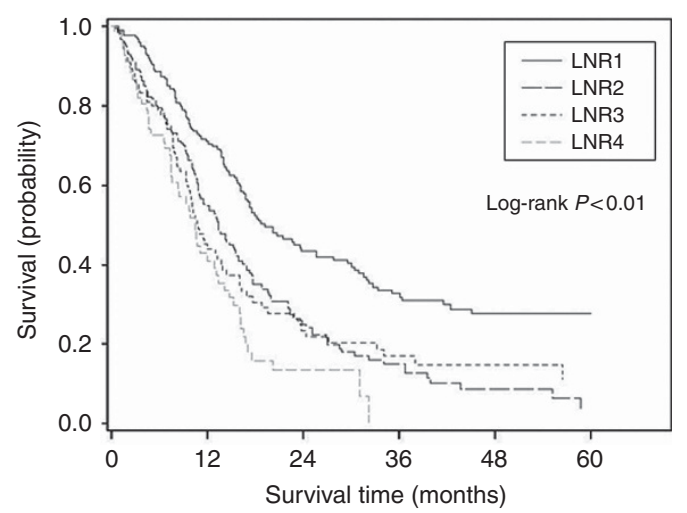

No. at risk

$\begin{array}{ll}\text { LNR1 } & 186 \\ \text { LNR2 } & 187 \\ \text { LNR3 } & 111 \\ \text { LNR4 } & 74\end{array}$

$\begin{array}{ll}186 & 113 \\ 187 & 85 \\ 111 & 35\end{array}$

55
30
16
5

\begin{abstract}
38
14
\end{abstract}

38
14
9
0

22
5
4
0

B

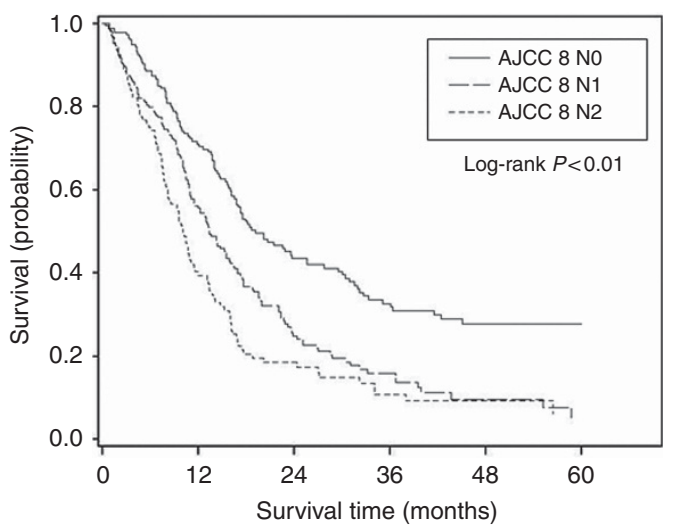

No. at risk

\begin{tabular}{lcccccc}
\hline AJCC 7 N0 & 186 & 113 & 55 & 38 & 22 & 16 \\
AJCC 8 N1 & 216 & 98 & 34 & 15 & 6 & 2 \\
AJCC 8 N2 & 156 & 45 & 17 & 8 & 3 & 1
\end{tabular}

D

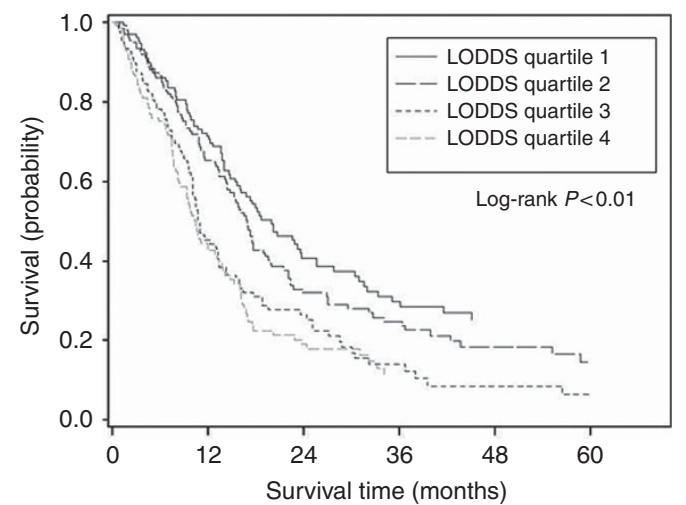

No. at risk

$\begin{array}{lllllll}\text { LODDS1 } & 139 & 85 & 36 & 23 & 13 & 7\end{array}$

$\begin{array}{lllllll}\text { LODDS2 } & 138 & 80 & 34 & 23 & 11 & 7\end{array}$

$\begin{array}{lllllll}\text { LODDS3 } & 141 & 47 & 20 & 8 & 4 & 3\end{array}$

$\begin{array}{lllllll}\text { LODDS4 } & 140 & 44 & 16 & 7 & 3 & 2\end{array}$

Figure 1. Kaplan-Meier curves for disease-free survival. (A) American Joint Committee on Cancer (AJCC) 7th edition. (B) American Joint Committee on Cancer 8th edition. (C) Lymph node ratio (LNR). LNR1: LNR=0; LNR2: $0<L N R \leqslant 0.2$; LNR3: $0.2<L N R \leqslant 0.4$; LNR4: LNR $>0.4$. (D) Quartiles of log(e) odds of metastatic lymph nodes (LODDS).

role of LN metastases in patients undergoing distal pancreatic resections for PDAC is not well characterised (Basturk et al, 2015; Malleo et al, 2015; Strobel et al, 2015). Our findings highlight the need to evaluate the prognostic role of $\mathrm{LN}$ involvement in surgical series with distal resections and margin-positive resections and should be interpreted cautiously, given the potential for diminished statistical power in stratified analyses.

We examined two other LN classification approaches, LNR and LODDS, which incorporate the total number of LN examined. Single-centre and population-based studies have found that LNR is a significant predictor of survival in resected PDAC (Slidell et al, 2008; Valsangkar et al, 2013). In our data, we saw little evidence for improved prognostication using LNR compared with AJCC (8th edition); this may partly be due to a relatively high number of LN harvested in our patient population. However, it has been unclear whether the prognostic value of LNR is due to accounting for misclassification, where fewer LN examined by the pathologist leads to lower likelihood of finding involved $\mathrm{LN}$ and an inappropriate $\mathrm{pN}$ stage, or better surgical technique, where more $\mathrm{LN}$ are harvested leading to fewer involved LN remaining in the patient.

The LODDS classification has also been recently evaluated in PDAC (National Comprehensive Cancer Network; Riediger et al, 2016). A proposed advantage of this parameter is that it assigns a value to LN-negative patients based on the number of examined LN. Therefore, it is designed to risk stratify patients with both LNnegative and -positive tumours. In the current study, no advantage to this classification approach was evident when divided into four exposure groups by quartiles. Overall, our data suggest that the AJCC (8th edition) classification adds value to prognostication while only modestly increasing complexity for practicing clinicians.

We observed that the presence of LVI was associated with a trend towards poor recurrence and survival outcomes in both LNnegative and -positive cases. As LVI has been associated with higher risk of disease recurrence and mortality (Takahashi et al, 2012), these results suggest that LVI may be considered regardless of LN status.

Recent ASCO clinical practice guidelines for potentially curable pancreatic cancer recommended adjuvant chemoradiation for patients with R1 resections or LN-positive disease (Khorana et al, 2016). The primary aim of adjuvant radiation therapy is to prevent local recurrence around the pancreatic resection bed, ultimately aiming for reduced local symptoms and improved survival. However, studies have been inconclusive regarding the benefits of adjuvant radiation (Neoptolemos et al, 2004; Regine et al, 2011), leaving clinicians and patients with a difficult choice regarding inclusion of chemoradiation in adjuvant treatment programmes. In our patient population, we found no evidence 
Table 4. Disease-free survival and overall survival by lymph node classification stratified by resection margin status and pancreatic resection type

\begin{tabular}{|c|c|c|c|c|c|c|c|c|c|c|}
\hline & \multicolumn{5}{|c|}{ Disease-free survival } & \multicolumn{5}{|c|}{ Overall survival } \\
\hline & $\begin{array}{c}\text { Number } \\
\text { of } \\
\text { patients }\end{array}$ & $\mathrm{HR}^{\mathrm{a}}(95 \% \mathrm{Cl})$ & $\begin{array}{c}\text { Number } \\
\text { of } \\
\text { patients }\end{array}$ & $\operatorname{HR}^{a}(95 \% \mathrm{Cl})$ & $P_{\text {interaction }}$ & $\begin{array}{c}\text { Number } \\
\text { of } \\
\text { patients }\end{array}$ & $\mathrm{HR}^{\mathrm{a}}(95 \% \mathrm{Cl})$ & $\begin{array}{c}\text { Number } \\
\text { of } \\
\text { patients }\end{array}$ & $\operatorname{HR}^{a}(95 \% \mathrm{Cl})$ & $P_{\text {interaction }}$ \\
\hline \multicolumn{11}{|c|}{ AJCC (7th edition) } \\
\hline pNO & 121 & 1.00 (reference) & 63 & 1.00 (reference) & 0.016 & 121 & 1.00 (reference) & 64 & 1.00 (reference) & 0.248 \\
\hline \multicolumn{11}{|c|}{ AJCC (8th edition) } \\
\hline pNO & 121 & 1.00 (reference) & 63 & 1.00 (reference) & \multirow[t]{3}{*}{0.005} & 121 & 1.00 (reference) & 64 & 1.00 (reference) & \multirow[t]{3}{*}{0.233} \\
\hline pN1 & 108 & $1.91(1.33-2.74)$ & 107 & $1.20(0.82-1.74)$ & & 109 & $1.94(1.35-2.77)$ & 107 & $1.51(1.01-2.25)$ & \\
\hline \multirow[t]{2}{*}{ pN2 } & 62 & $2.75(1.82-4.17)$ & 94 & $1.59(1.06-2.40)$ & & 63 & $2.44(1.63-3.66)$ & 95 & $1.95(1.28-2.99)$ & \\
\hline & \multicolumn{2}{|c|}{ Pancreaticoduodenectomy } & \multicolumn{2}{|c|}{ Distal resection } & $\boldsymbol{P}_{\text {interaction }}\lceil$ & \multicolumn{2}{|c|}{ Pancreaticoduodenectomy } & \multicolumn{2}{|c|}{ Distal resection } & $P_{\text {interaction }}$ \\
\hline pNO & 115 & 1.00 (reference) & 67 & 1.00 (reference) & \multirow[t]{2}{*}{0.179} & 117 & 1.00 (reference) & 71 & 1.00 (reference) & \multirow[t]{2}{*}{0.022} \\
\hline pN1 & 300 & $2.13(1.58-2.89)$ & 64 & $1.51(0.94-2.43)$ & & 305 & $2.13(1.57-2.87)$ & 65 & $1.23(0.77-1.98)$ & \\
\hline \multicolumn{11}{|c|}{ AJCC (8th edition) } \\
\hline pNO & 115 & 1.00 (reference) & 67 & 1.00 (reference) & \multirow[t]{3}{*}{0.116} & 117 & 1.00 (reference) & 71 & 1.00 (reference) & \multirow[t]{3}{*}{0.003} \\
\hline pN1 & 161 & $1.85(1.34-2.56)$ & 49 & $1.53(0.94-2.49)$ & & 164 & $1.85(1.34-2.56)$ & 50 & $1.37(0.82-2.28)$ & \\
\hline pN2 & 139 & $2.63(1.87-3.70)$ & 15 & $1.42(0.60-3.36)$ & & 141 & $2.56(1.84-3.57)$ & 15 & $0.89(0.40-1.97)$ & \\
\hline
\end{tabular}

Table 5. Odds ratios for local recurrence by AJCC (7th and 8th edition) lymph node classification

\begin{tabular}{|l|c|c|c|}
\hline \multicolumn{3}{|c|}{} & \multicolumn{3}{c|}{ Any local recurrence $^{\text {a }}$} \\
\cline { 2 - 4 } \multicolumn{1}{|c|}{} & $\begin{array}{c}\text { Number of } \\
\text { cases }\end{array}$ & OR $^{\text {b }}$ (95\% Cl) & $P_{\text {trend }}$ \\
\hline AJCC (7th edition) \\
\hline pN0 & 110 & 1.00 (reference) & 0.472 \\
pN1 & 262 & $0.84(0.52-1.36)$ & \\
\hline AJCC (8th edition) \\
\hline pN0 & 110 & 1.00 (reference) & 0.815 \\
pN1 & 151 & $0.76(0.45-1.29)$ & \\
pN2 & 110 & $0.99(0.56-1.75)$ & \\
\hline
\end{tabular}

Abbreviations: $\mathrm{AJCC}=$ American Joint Committee on Cancer; $\mathrm{OR}=$ odds ratio.

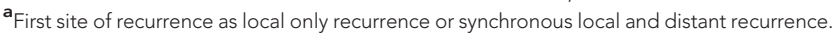

bodds ratio adjusted for age, sex, tumour grade, perioperative systemic chemotherapy, perioperative radiation therapy, resection margins, and institution.

that nodal metastases increased the risk for local disease as first site of recurrence. Although our sample sizes were reduced when stratified by treatment, this finding was consistent in patients who received perioperative radiation and those who did not. Therefore, data from our population would not support selecting patients for adjuvant chemoradiation based on nodal status, if the primary objective is to reduce local recurrence rates. The ongoing RTOG 0848 study will provide valuable information regarding the benefits of adjuvant chemoradiation.

The current study has several important strengths. Our patient population was derived from three US academic institutions in distinct geographic regions, as well as 35 other academic and community centres across the country. Patients were sequentially captured and selected based only on having undergone surgical resection. Thus, the study population represents a diversity of patient characteristics, surgical and pathological practices, and treatment approaches. Moreover, we had direct access to medical records and follow-up information, allowing for consistent and detailed data abstraction, including patterns of disease recurrence. These data also allowed for appropriate adjustment for confounding covariates in multivariate models and stratified analyses by other important characteristics, such as resection margin status. Last, we used training and validation sets to evaluate the performance of the different LN classification parameters and compared them with model discrimination and calibration metrics.

Study limitations also deserve mention. We included patients who received neoadjuvant therapy in our main analyses, and LN status may have been affected by preoperative treatment. However, sensitivity analyses confirmed highly similar results after excluding patients who received neoadjuvant therapy. The study period was not uniform across the contributing centres. However, adjuvant therapy programmes have not changed significantly during the follow-up time of the study, with most patients receiving adjuvant 5 -fluorouracil or adjuvant gemcitabine. Notably, the ESPAC-3 study demonstrated that patient outcomes are nearly identical with 5 -fluorouracil or gemcitabine delivered after surgical resection (Neoptolemos et al, 2010). We did not centrally rereview pathological specimens for resection margins and LVI, and therefore, our findings represent the information that is available for practicing clinicians in the real-world setting. Additional studies to compare LVI and resection margins across centres and pathologists would be of interest. Finally, follow-up of patients after surgical resection was performed as per standard institutional practice. If postoperative imaging was performed more frequently 
for patients with LN-positive disease, then this could accentuate differences in DFS time between the LN-positive and -negative groups. Nevertheless, no standard follow-up programme has been defined to improve outcomes in patients with resected PDAC, and imaging intervals would not be expected to alter overall survival times, which were markedly different between the LN-positive and -negative groups.

In conclusion, we confirmed the prognostic importance of regional LN metastases after resection for PDAC in a large, multiinstitutional database including patients from across the United States. In this patient population, the upcoming AJCC (8th edition) staging system is an effective and practical classification of regional LN involvement that incorporates the number of metastatic LN as a prognostic factor in the prediction of disease recurrence and survival. Nevertheless, its prognostic value may be weaker in patients with positive resection margins and in patients with distal resections. Notably, outcomes for patients with LN-positive disease were extremely poor. Novel approaches to treatment of this disease are greatly needed, including consideration to preoperative treatment, multiagent chemotherapy programmes, and therapeutic approaches based on an improved biologic understanding of this highly lethal malignancy (Perez et al, 2016; Ying et al, 2016; Neoptolemos et al, 2017).

\section{ACKNOWLEDGEMENTS}

This work was supported by NIH K07 CA148894 (to KN); by NCI R35 CA197735 (to SO); by the Robert T and Judith B Hale Fund for Pancreatic Cancer, Perry S Levy Fund for Gastrointestinal Cancer Research, Pappas Family Research Fund for Pancreatic Cancer, NIH R01 CA124908, and NIH P50 CA127003 (to CSF); MyBlueDots Fund (to ACK); and by NIH/NCI U01 CA210171, Department of Defense CA130288, Lustgarten Foundation, Pancreatic Cancer Action Network, Noble Effort Fund, Peter R Leavitt Family Fund, Wexler Family Fund, and Promises for Purple (to BMW).

\section{CONFLICT OF INTEREST}

The authors declare no conflict of interest.

\section{REFERENCES}

National Comprehensive Cancer Network. Pancretic adenocarcinoma. Available at: https://www.nccn.org/professionals/physician_gls/ f_guidelines.asp.

Allen PJ, Kuk D, Castillo CF, Basturk O, Wolfgang CL, Cameron JL, Lillemoe KD, Ferrone CR, Morales-Oyarvide V, He J, Weiss MJ, Hruban RH, Gonen M, Klimstra DS, Mino-Kenudson M (2017) MultiInstitutional Validation Study of the American Joint Commission on Cancer (8th Edition) changes for $\mathrm{T}$ and $\mathrm{N}$ staging in patients with pancreatic adenocarcinoma. Ann Surg 265(1): 185-191.

Basturk O, Saka B, Balci S, Postlewait LM, Knight J, Goodman M, Kooby D, Sarmiento JM, El-Rayes B, Choi H, Bagci P, Krasinskas A, Quigley B, Reid MD, Akkas G, Maithel SK, Adsay V (2015) Substaging of lymph node status in resected pancreatic ductal adenocarcinoma has strong prognostic correlations: proposal for a revised $\mathrm{N}$ classification for TNM staging. Ann Surg Oncol 22(Suppl 3): S1187-S1195.

Demler OV, Paynter NP, Cook NR (2015) Tests of calibration and goodnessof-fit in the survival setting. Stat Med 34(10): 1659-1680.

Edge S, Byrd DR, Compton CC, Fritz AG, Greene FL, Trotti A (2010) AJCC Cancer Staging Manual. Springer: New York, NY, USA.

Hellan M, Sun CL, Artinyan A, Mojica-Manosa P, Bhatia S, Ellenhorn JD, Kim J (2008) The impact of lymph node number on survival in patients with lymph node-negative pancreatic cancer. Pancreas 37(1): 19-24.
Khorana AA, Mangu PB, Berlin J, Engebretson A, Hong TS, Maitra A, Mohile SG, Mumber M, Schulick R, Shapiro M, Urba S, Zeh HJ, Katz MH (2016) Potentially curable pancreatic cancer: American Society of Clinical Oncology Clinical Practice Guideline. J Clin Oncol 34(21): 2541-2556.

La Torre M, Nigri G, Petrucciani N, Cavallini M, Aurello P, Cosenza G, Balducci G, Ziparo V, Ramacciato G (2014) Prognostic assessment of different lymph node staging methods for pancreatic cancer with R0 resection: $\mathrm{pN}$ staging, lymph node ratio, log odds of positive lymph nodes. Pancreatology 14(4): 289-294.

Lim JE, Chien MW, Earle CC (2003) Prognostic factors following curative resection for pancreatic adenocarcinoma: a population-based, linked database analysis of 396 patients. Ann Surg 237(1): 74-85.

Malleo G, Maggino L, Capelli P, Gulino F, Segattini S, Scarpa A, Bassi C, Butturini G, Salvia R (2015) Reappraisal of nodal staging and study of lymph node station involvement in pancreaticoduodenectomy with the standard international study group of pancreatic surgery definition of lymphadenectomy for cancer. J Am Coll Surg 221(2): 367-79 e4.

Murakami Y, Uemura K, Hayashidani Y, Sudo T, Ohge H, Sueda T (2007) Pancreatoduodenectomy for distal cholangiocarcinoma: prognostic impact of lymph node metastasis. World J Surg 31(2): 337-342, discussion 343-344.

Neoptolemos JP, Palmer DH, Ghaneh P, Psarelli EE, Valle JW, Halloran CM, Faluyi O, O'Reilly DA, Cunningham D, Wadsley J, Darby S, Meyer T, Gillmore R, Anthoney A, Lind P, Glimelius B, Falk S, Izbicki JR, Middleton GW, Cummins S, Ross PJ, Wasan H, McDonald A, Crosby T, Ma YT, Patel K, Sherriff D, Soomal R, Borg D, Sothi S, Hammel P, Hackert T, Jackson R, Buchler MW, European Study Group for Pancreatic C (2017) Comparison of adjuvant gemcitabine and capecitabine with gemcitabine monotherapy in patients with resected pancreatic cancer (ESPAC-4): a multicentre, open-label, randomised, phase 3 trial. Lancet 38(10073): 1011-1024.

Neoptolemos JP, Stocken DD, Bassi C, Ghaneh P, Cunningham D, Goldstein D, Padbury R, Moore MJ, Gallinger S, Mariette C, Wente MN, Izbicki JR, Friess H, Lerch MM, Dervenis C, Olah A, Butturini G, Doi R, Lind PA, Smith D, Valle JW, Palmer DH, Buckels JA, Thompson J, McKay CJ, Rawcliffe CL, Buchler MW, European Study Group for Pancreatic C (2010) Adjuvant chemotherapy with fluorouracil plus folinic acid vs gemcitabine following pancreatic cancer resection: a randomized controlled trial. JAMA 304(10): 1073-1081.

Neoptolemos JP, Stocken DD, Friess H, Bassi C, Dunn JA, Hickey H, Beger H, Fernandez-Cruz L, Dervenis C, Lacaine F, Falconi M, Pederzoli P, Pap A, Spooner D, Kerr DJ, Buchler MW, European Study Group for Pancreatic C (2004) A randomized trial of chemoradiotherapy and chemotherapy after resection of pancreatic cancer. N Engl J Med 350(12): $1200-1210$.

Oettle H, Neuhaus P, Hochhaus A, Hartmann JT, Gellert K, Ridwelski K, Niedergethmann M, Zulke C, Fahlke J, Arning MB, Sinn M, Hinke A, Riess H (2013) Adjuvant chemotherapy with gemcitabine and long-term outcomes among patients with resected pancreatic cancer: the CONKO001 randomized trial. JAMA 310(14): 1473-1481.

Oettle H, Post S, Neuhaus P, Gellert K, Langrehr J, Ridwelski K, Schramm H, Fahlke J, Zuelke C, Burkart C, Gutberlet K, Kettner E, Schmalenberg H, Weigang-Koehler K, Bechstein WO, Niedergethmann M, Schmidt-Wolf I, Roll L, Doerken B, Riess H (2007) Adjuvant chemotherapy with gemcitabine vs observation in patients undergoing curative-intent resection of pancreatic cancer: a randomized controlled trial. JAMA 297(3): 267-277.

Pencina MJ, D'Agostino RB (2004) Overall C as a measure of discrimination in survival analysis: model specific population value and confidence interval estimation. Stat Med 23(13): 2109-2123.

Perez K, Clancy TE, Mancias JD, Rosenthal MH, Wolpin BM (2016) When, what, and why of perioperative treatment of potentially curable pancreatic adenocarcinoma. J Clin Oncol; epub ahead of print 28 December 2016; doi:JCO2016702134.

Regine WF, Winter KA, Abrams R, Safran H, Hoffman JP, Konski A, Benson AB, Macdonald JS, Rich TA, Willett CG (2011) Fluorouracil-based chemoradiation with either gemcitabine or fluorouracil chemotherapy after resection of pancreatic adenocarcinoma: 5-year analysis of the US Intergroup/RTOG 9704 phase III trial. Ann Surg Oncol 18(5): 1319-1326.

Regine WF, Winter KA, Abrams RA, Safran H, Hoffman JP, Konski A, Benson AB, Macdonald JS, Kudrimoti MR, Fromm ML, Haddock MG, Schaefer P, Willett CG, Rich TA (2008) Fluorouracil vs gemcitabine chemotherapy before and after fluorouracil-based chemoradiation 
following resection of pancreatic adenocarcinoma: a randomized controlled trial. JAMA 299(9): 1019-1026.

Richter A, Niedergethmann M, Sturm JW, Lorenz D, Post S, Trede M (2003) Long-term results of partial pancreaticoduodenectomy for ductal adenocarcinoma of the pancreatic head: 25-year experience. World J Surg 27(3): 324-329.

Riediger H, Kulemann B, Wittel U, Adam U, Sick O, Neeff H, Hoppner J, Hopt UT, Makowiec F (2016) Prognostic role of log odds of lymph nodes after resection of pancreatic head cancer. J Gastrointest Surg 20(10): 1707-1715.

Schwarz RE, Smith DD (2006) Extent of lymph node retrieval and pancreatic cancer survival: information from a large US population database. Ann Surg Oncol 13(9): 1189-1200.

Siegel RL, Miller KD, Jemal A (2016) Cancer statistics, 2016. CA Cancer J Clin 66(1): 7-30.

Slidell MB, Chang DC, Cameron JL, Wolfgang C, Herman JM, Schulick RD, Choti MA, Pawlik TM (2008) Impact of total lymph node count and lymph node ratio on staging and survival after pancreatectomy for pancreatic adenocarcinoma: a large, population-based analysis. Ann Surg Oncol 15(1): 165-174.

Strobel O, Hinz U, Gluth A, Hank T, Hackert T, Bergmann F, Werner J, Buchler MW (2015) Pancreatic adenocarcinoma: number of positive nodes allows to distinguish several N categories. Ann Surg 261(5): 961-969.

Takahashi H, Ohigashi H, Ishikawa O, Gotoh K, Yamada T, Nagata S, Tomita Y, Eguchi H, Doki Y, Yano M (2012) Perineural invasion and lymph node involvement as indicators of surgical outcome and pattern of recurrence in the setting of preoperative gemcitabine-based chemoradiation therapy for resectable pancreatic cancer. Ann Surg 255(1): 95-102.

Valsangkar NP, Bush DM, Michaelson JS, Ferrone CR, Wargo JA, Lillemoe KD, Fernandez-del Castillo C, Warshaw AL, Thayer SP (2013) N0/N1, PNL, or LNR? The effect of lymph node number on accurate survival prediction in pancreatic ductal adenocarcinoma. J Gastrointest Surg 17(2): 257-266.

Winter JM, Cameron JL, Campbell KA, Arnold MA, Chang DC, Coleman J, Hodgin MB, Sauter PK, Hruban RH, Riall TS, Schulick RD, Choti MA, Lillemoe KD, Yeo CJ (2006) 1423 pancreaticoduodenectomies for pancreatic cancer: A single-institution experience. J Gastrointest Surg 10(9): 1199-1210, discussion 1210-1211.

Ying H, Dey P, Yao W, Kimmelman AC, Draetta GF, Maitra A, DePinho RA (2016) Genetics and biology of pancreatic ductal adenocarcinoma. Genes Dev 30(4): 355-385.

This work is published under the standard license to publish agreement. After 12 months the work will become freely available and the license terms will switch to a Creative Commons AttributionNonCommercial-Share Alike 4.0 Unported License.

Supplementary Information accompanies this paper on British Journal of Cancer website (http://www.nature.com/bjc) 